\title{
Nutritional evaluation study based on NRS 2002, OPNI, and their combined use in patients with adverse drug reactions after chemotherapy: a cross-sectional study
}

\author{
Yun Bian ${ }^{1}$, Fen Xie ${ }^{1}$, Jianbo Han ${ }^{2}$, Yongjuan Ding ${ }^{1}$ \\ ${ }^{1}$ Department of Pharmacy, Affiliated Hospital of Jiangnan University, Wuxi, China; ${ }^{2}$ Department of Neurosurgery, Wujin Hospital Affiliated to \\ Jiangsu University, Changzhou, China \\ Contributions: (I) Conception and design: Y Bian; (II) Administrative support: Y Ding; (III) Provision of study materials or patients: F Xie; (IV) \\ Collection and assembly of data: J Han; (V) Data analysis and interpretation: Y Bian; (VI) Manuscript writing: All authors; (VII) Final approval of \\ manuscript: All authors. \\ Correspondence to: Yongjuan Ding. 1000 Hefeng Road, Wuxi 214000, China. Email: 2649570095@163.com.
}

Background: It is important to assess the nutritional status of patients who have experienced adverse drug reactions (ADRs) after chemotherapy. We aimed to explore the nutritional status of patients who developed ADRs after chemotherapy, using the Nutritional Risk Screening 2002 (NRS 2002) tool, the Onodera Prognostic Nutrition Index (OPNI), and their combined application.

Methods: NRS 2002 screening and OPNI calculation for patients before chemotherapy. Patients with ADRs after chemotherapy were grouped according to the NRS 2002, OPNI, and combined scores from both assessments. The types of ADRs were classified according to the National adverse drug reaction monitoring system (http://www.adrs.org.cn/). The impact of nutritional risk on the classification and types of ADRs in cancer chemotherapy patients was analyzed. Logistic regression was used to analyze the key influencing factors of gastrointestinal damage and bone marrow suppression. the consistency between the NRS 2002, OPNI, and their combined application analyzed.

Results: The difference in body mass index (BMI) scores between the OPNI (P=0.041) and NRS 2002 groups was statistically significant $(\mathrm{P}=0.051)$. The difference in ADR type in the OPNI subgroups $(\mathrm{P}=0.04)$ was statistically significant. It showed that the proportion of new and severe ADRs in the low OPNI group (47.14\%) was significantly higher than that in the high OPNI group (27.13\%). The differences in digestive tract-associated ADRs were statistically significant among the OPNI groups ( $\mathrm{P}=0.004)$, NRS 2002 groups $(\mathrm{P}=0.012)$, and combined measures groups $(\mathrm{P}=0.000)$, as were the differences in myelosuppressive-type ADRs in the OPNI groups $(\mathrm{P}=0.035)$, NRS 2002 groups $(\mathrm{P}=0.000)$, and combined measures groups $(\mathrm{P}=0.000)$. Logistic regression analysis showed that $\mathrm{BMI}$ was the key influencing factor for digestive tract-associated ADRs (95\% CI: 1.267, 95\% CI: 1.022-1.570, P=0.031) and myelosuppressive-type ADRs (95\% CI: 1.213, 95\% CI: $1.020-1.443, \mathrm{P}=0.029)$. It had good consistency with the combined measures of nutritional risk (Kappa value $=0.675)$.

Conclusions: Patients with severe ADRs after chemotherapy showed low OPNI values, high NRS 2002 scores, and malnutrition. These patients also had a significantly increased incidence of digestive tract and myelosuppressive-type ADRs with BMI as the key influencing factor. The combined assessments showed good consistency with the NRS 2002 scores.

Keywords: Nutritional Risk Screening 2002 (NRS 2002); Onodera Prognostic Nutrition Index (OPNI); combined application; chemotherapy; adverse drug reaction (ADR)

Submitted Nov 30, 2021. Accepted for publication Jan 27, 2022.

doi: $10.21037 /$ atm-22-256

View this article at: https://dx.doi.org/10.21037/atm-22-256 


\section{Introduction}

Cancer is a worldwide major public health problem. According to the statistics (1), there are 18.1 million new cancer cases and 9.6 million deaths globally every year. Chemotherapy is the most common treatment for cancer patients, with significant clinical efficacy. However, because chemotherapy is not targeted, it kills cancer cells and damages normal cells, leading to many adverse reactions and complications that seriously affect the therapeutic effect and the health of patients. Adverse drug reactions (ADRs) are defined as harmful reactions to prescribed drugs that have nothing to do with the purpose of the drug use under normal usage and dosage. The occurrence of ADRs, especially severe ADRs, will force changes to first-line chemotherapy regimens, prolong the time of chemotherapy, or even interrupt chemotherapy, thus affecting the prognosis of patients. Clinical practice has found that malnourished cancer patients are more prone to experience ADRs during chemotherapy. An Onodera Prognostic Nutrition Index (OPNI) value is easy to obtain and is generally used to predict the occurrence of postoperative complications and the prognosis of advanced cancer patients. The Nutritional Risk Screening 2002 (NRS 2002) tool is commonly used for nutritional risk assessment and screening of hospitalized patients. However, few studies have examined their use and evaluated their association with the type and classification of ADRs in patients. Therefore, this study innovatively assessed the nutritional status of patients with ADRs to explore the clinical value of OPNI and NRS 2002 nutritional risk screening and their combined use in evaluating the occurrence (risk, type, etc.) of ADRs in chemotherapy patients so as to reduce the occurrence of ADRs and improve the compliance of patients with medication. In addition, this study used OPNI, NRS 2002, and a combination of both measures for the statistical analyses. This research innovatively combined the selected nutritional screening evaluation methods and verified this combined form statistically. Our aim in using the combined measures approach was to improve the accuracy and relevance of nutritional screening evaluations and identify patients who are likely to benefit from more targeted nutritional support interventions, consistent with China's health insurance policy. The cases involved in this study are from the Chinese Hospital Pharmacovigilance System (http://www.adrs.org.cn/). These cases contain different tumor types, and the investigator has not identified a certain case for the study. In the follow-up research process, consider identifying to choose a specific type of cancer patient for research.

We present the following article in accordance with the STROBE reporting checklist (available at https://atm. amegroups.com/article/view/10.21037/atm-22-256/rc).

\section{Methods}

\section{Case data}

This study selected 199 cancer patients with ADRs who received chemotherapy at the Affiliated Hospital of Jiangnan University, China, from January 2019 to December 2020. The ADRs were identified by the Chinese Hospital Pharmacovigilance System (http://www.adrs.org.cn/). The patients included 100 males and 99 females. Among them, there were 69 cases of gastrointestinal cancer, 32 cases of lung cancer, 26 cases of hematologic cancers, 16 cases of gynecological cancers, 16 cases of breast cancer, 9 cases of head and neck cancer, 6 cases of pancreatic cancer, 3 cases of liver cancer, and 22 cases of other cancers. All procedures performed in this study involving human participants were in accordance with the Declaration of Helsinki (as revised in 2013). The study was approved by ethics board of Affiliated Hospital of Jiangnan University (No. LS2020034). Individual consent for this retrospective analysis was waived.

\section{Related definitions}

The OPNI value was calculated according to the patients' routine blood and biochemical examination results before chemotherapy, where the OPNI value $=$ albumin value $(\mathrm{g} / \mathrm{L})$ $+5 \times$ total lymphocyte number $\left(10^{9} / \mathrm{L}\right)$. According to the criteria developed by Cai et al. (2), the low OPNI group was defined by an OPNI value $<45.8$, and the high OPNI group was defined by an OPNI value $\geq 45$. 8 . The NRS 2002 was developed by the European Society for Parenteral and Enteral Nutrition (ESPEN) in 2002 and is recommended for nutritional risk screening in adult hospitalized patients. The score is the sum of the nutritional status score, disease severity score, and age score, and a total score $\geq 3$ indicates the patient is at nutritional risk. Therefore, the low NRS 2002 group was defined by a score $<3$, and the high NRS 2002 group was defined by a score $\geq 3$. The combined 'good nutrition' group was defined by an OPNI value $\geq 45.8$ and NRS 2002 score $<3$, and the combined 'poor nutrition' group was defined by an OPNI value $<45.8$ and NRS 2002 score $\geq 3$. 
Table 1 Clinical characteristics comparison of the OPNI, NRS 2002, and combined assessment groups

\begin{tabular}{|c|c|c|c|c|c|c|c|c|c|}
\hline Variables & $\begin{array}{l}\text { OPNI low } \\
\text { group }(n=70)\end{array}$ & $\begin{array}{c}\text { OPNI high } \\
\text { group }(n=129)\end{array}$ & $P$ value & $\begin{array}{l}\text { NRS } 2002 \text { low } \\
\text { group }(n=159)\end{array}$ & $\begin{array}{l}\text { NRS } 2002 \text { high } \\
\text { group }(n=40)\end{array}$ & P value & $\begin{array}{c}\text { Combined } \\
\text { good nutrition } \\
\text { group }(n=177)\end{array}$ & $\begin{array}{c}\text { Combined } \\
\text { poor nutrition } \\
\text { group }(n=22)\end{array}$ & $P$ value \\
\hline Male & 35 & 65 & & 79 & 21 & & 86 & 16 & \\
\hline Female & 35 & 64 & & 80 & 19 & & 89 & 8 & \\
\hline $\mathrm{BMI}\left(\mathrm{kg} / \mathrm{m}^{2}, \bar{x} \pm \mathrm{s}\right)$ & $23.63 \pm 3.71$ & $22.35 \pm 3.65$ & 0.041 & $23.03 \pm 3.62$ & $21.88 \pm 3.97$ & 0.051 & $22.68 \pm 3.70$ & $23.75 \pm 3.74$ & 0.262 \\
\hline ADR type & & & 0.04 & & & 0.874 & & & 0.224 \\
\hline General & 37 & 94 & & 105 & 26 & & 119 & 12 & \\
\hline
\end{tabular}

OPNI, Onodera Prognostic Nutrition Index; NRS 2002, Nutritional Risk Screening 2002; BMI, body mass index.

Adverse Drug Reaction Report and Monitoring Measures for The Administration (3) define new ADRs in bylaws section: (I) no such adverse reactions in the leaflet; (II) prospectus has been described, but the nature of the adverse reactions occur, the degree, the consequences or frequency do not agree with manual description or more serious, according to the new adverse drug reactions; serious ADR: refers to use the drug caused the damage under the circumstances of the reaction, including the following situations: the cause of death; life-threatening; carcinogenic, teratogenic and cause birth defects; lead to significant or permanent disability or organs injury; lead to prolonged hospitalization, or hospital; lead to other important medical event, may be listed in the above case without treatment.

\section{Statistical analysis}

We retrospectively checked the patient's routine blood, liver, and kidney function test results acquired before chemotherapy in the hospital medical record system (MandalaT Software Corporation) and calculated the OPNI value. We also collected the NRS 2002 score recorded by nursing staff prior to chemotherapy (MandalaT Software Corporation). Patients were grouped according to the OPNI value, NRS 2002 score, and a combination of scores from both measures. By definition, cases were divided into low versus high OPNI groups, low versus high NRS 2002 groups, and a combined 'good nutrition' group versus a combined 'poor nutrition' group.
SSPS v.26.0 software was used for the analysis. Normality and homogeneity of variance tests were carried out, and measurement data were described by the mean and standard deviation $(\bar{x} \pm s)$. The Wilcoxon rank-sum test was used to compare univariate groups that were not normally distributed, whereas the $t$-test was used to compare normally distributed univariate groups. The $\chi^{2}$ test was used for comparison of categorical variables between groups. A multivariate logistic regression analysis was used to assess the key influencing clinical variables associated with two significant ADRs: digestive tract damage and myelosuppression. A $\mathrm{P}$ value $<0.05$ was considered statistically significant.

\section{Results}

Comparison of clinical data between the OPNI, NRS 2002, and combined assessment groups

This study retrospectively analyzed 199 patients with ADRs acquired after chemotherapy from January 2019 to December 2020. Patients were grouped according to the different nutritional risk screening assessment methods, and the differences in clinical characteristics between the groups were compared (Table 1). The difference in BMI between the OPNI group and the NRS 2002 group was statistically significant. There was no statistically significant difference in BMI among the combined assessment groups. In addition, the difference in ADR types in the OPNI group was statistically significant. The proportion of new 
Table 2 Comparison of ADR classifications between the OPNI, NRS 2002, and combined application groups

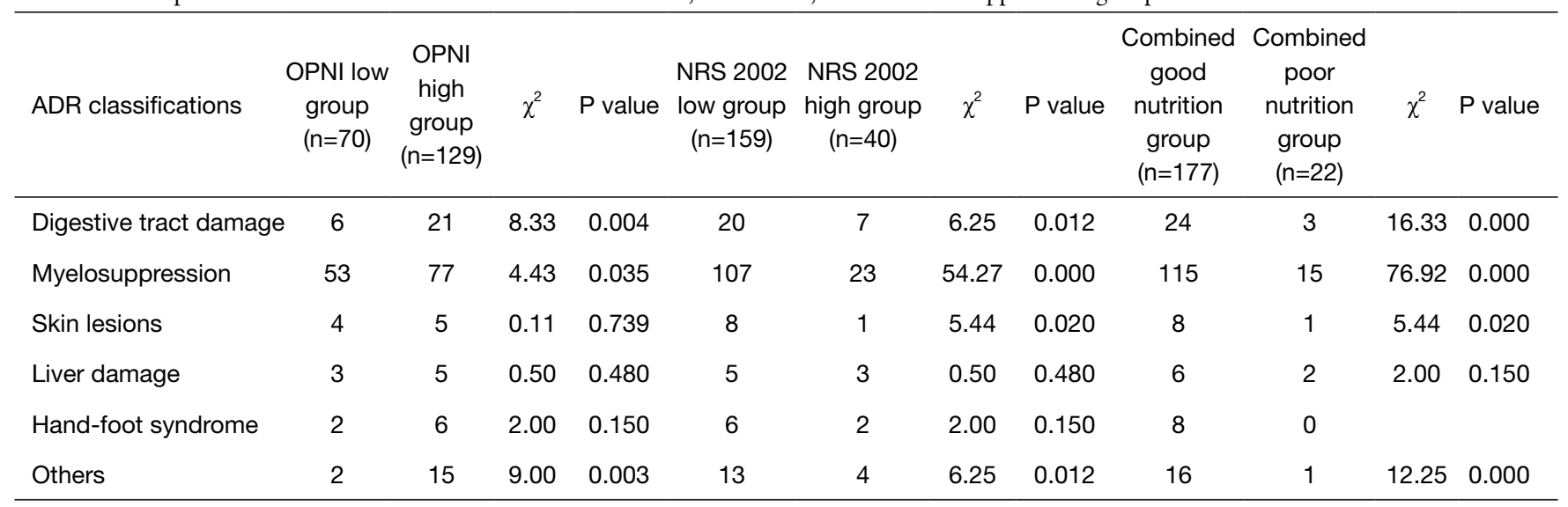

OPNI, Onodera Prognostic Nutrition Index; NRS 2002, Nutritional Risk Screening 2002; ADR, adverse drug reaction.

Table 3 Logistic regression analysis of clinical characteristics and digestive tract damage

\begin{tabular}{lccc}
\hline Clinical characteristics & EXP(B) & $95 \% \mathrm{Cl}$ & P value \\
\hline BMI & 1.267 & $1.022-1.570$ & 0.031 \\
Gender & 1.455 & $0.335-6.322$ & 0.617 \\
Age & 1.014 & $0.954-1.077$ & 0.655 \\
NRS 2002 & 2.094 & $0.239-18.332$ & 0.504 \\
OPNI & 1.368 & $0.184-10.180$ & 0.760 \\
Combined assessment & 2.343 & $0.026-207.991$ & 0.710 \\
Metastasis & 1.450 & $0.371-5.662$ & 0.593 \\
Hemoglobin & 0.963 & $0.927-1.001$ & 0.054 \\
Complications & 1.616 & $0.400-6.526$ & 0.050 \\
\hline
\end{tabular}

OPNI, Onodera Prognostic Nutrition Index; NRS 2002, Nutritional Risk Screening 2002; BMI, body mass index; CI, confidence interval; EXP, expiry date.

and serious ADRs in the low OPNI group (47.14\%) was significantly higher than that in the high OPNI group $(27.13 \%)$. The difference in ADR types between the NRS 2002 and the combined assessment groups was not statistically significant. There was no statistically significant difference in gender or age.

\section{Comparison of ADR classification between the OPNI, NRS 2002, and combined assessment groups}

The 199 patients with ADRs after chemotherapy were compared according to the different ADR classifications.
Rates of digestive tract damage and myelosuppression were both significantly different in the OPNI, NRS 2002, and combined assessment subgroups. The differences between the OPNI, NRS 2002, and combined assessment subgroups in 'other' ADRs were also statistically significant. In this study, 'other' ADRs included nervous system damage, fever, chills, vasculitis, etc., which had many categories but few absolute cases, so an analysis of the individual ADRs could not be conducted. The difference between the NRS 2002 and combined assessment subgroups in skin lesion ADRs was statistically significant. There were no significant differences within the OPNI, NRS 2002, and combined assessment subgroups for liver damage or hand-foot syndrome ADRs. The results are shown in Table 2.

\section{Multivariate analysis of the occurrence of digestive tract damage}

In this study, a total of 27 patients developed ADRs involving digestive tract damage after chemotherapy. A multivariate logistic regression analysis was performed on the correlation between BMI, gender, age, NRS 2002, OPNI, combined assessment, presence or absence of metastasis, hemoglobin, complications, and the occurrence of digestive tract damage. The occurrence of digestive tract damage after chemotherapy was significantly correlated with BMI [EXP(B): 1.267, 95\% CI: 1.022-1.570, $\mathrm{P}=0.031]$, and a lower BMI was a high-risk factor for the occurrence of ADRs involving digestive tract damage after chemotherapy, as shown in Table 3. 
Table 4 Logistic regression analysis of clinical characteristics and myelosuppression

\begin{tabular}{lccc}
\hline Clinical characteristics & EXP(B) & $95 \% \mathrm{Cl}$ & $\mathrm{P}$ values \\
\hline BMI & 1.213 & $1.020-1.443$ & 0.029 \\
Gender & 1.354 & $0.437-4.194$ & 0.599 \\
Age & 1.009 & $0.963-1.057$ & 0.711 \\
NRS 2002 & 0.933 & $0.140-6.212$ & 0.943 \\
OPNI & 0.509 & $0.113-2.305$ & 0.381 \\
Combined assessment & 2.517 & $0.047-134.730$ & 0.649 \\
Metastasis & 2.219 & $0.782-6.295$ & 0.134 \\
Hemoglobin & 0.981 & $0.952-1.010$ & 0.202 \\
Complications & 1.038 & $0.329-3.279$ & 0.949
\end{tabular}

OPNI, Onodera Prognostic Nutrition Index; NRS 2002, Nutritional Risk Screening 2002; BMI, body mass index; Cl, confidence interval; EXP, expiry date.

Table 5 Analysis of consistency between NRS 2002 and OPNI diagnosis of nutritional risk

\begin{tabular}{lccc}
\hline \multirow{2}{*}{ Consistency } & \multicolumn{2}{c}{ OPNI } & Total \\
\cline { 2 - 3 } & Low group & High group & \\
\hline NRS 2002 & & & 160 \\
Low group & 48 & 112 & 39 \\
High group & 22 & 17 & 199 \\
Total & 70 & 129 & \\
Kappa value & & -0.141 & \\
T value & & -3.097 & \\
P value & & 0.002 & \\
\hline
\end{tabular}

OPNI, Onodera Prognostic Nutrition Index; NRS 2002, Nutritional Risk Screening 2002.

\section{Multivariate analysis of the occurrence of myelosuppression}

In this study, a total of 130 patients developed myelosuppression ADRs after chemotherapy. A multivariate logistic regression analysis was performed on the correlation between BMI, gender, age, NRS 2002, OPNI, combined assessment, presence or absence of metastasis, hemoglobin, complications, and the occurrence of myelosuppression. There was a significant correlation between the occurrence of myelosuppression after chemotherapy and BMI $[\mathrm{EXP}(\mathrm{B})$ : $1.213,95 \%$ CI: $1.020-1.443, \mathrm{P}=0.029]$. A low BMI was a high-risk factor for myelosuppression-type ADRs after
Table 6 Analysis of consistency between NRS 2002 and combined assessment diagnosis of nutritional risk

\begin{tabular}{lccc}
\hline \multirow{2}{*}{ Consistency } & \multicolumn{2}{c}{ Combined assessment } & Total \\
\cline { 2 - 3 } & Good & Poor & \\
\hline NRS 2002 & & & \\
Low group & 160 & 0 & 160 \\
High group & 17 & 22 & 39 \\
Total & 177 & 22 & 199 \\
Kappa value & & 0.675 & \\
T value & & 10.073 & \\
P value & & 0.000 & \\
\hline
\end{tabular}

NRS 2002, Nutritional Risk Screening 2002.

chemotherapy, as shown in Table 4.

\section{Consistency analysis between NRS 2002 and OPNI, and between NRS 2002 and the combined assessment}

NRS 2002 demonstrated a poor consistency with OPNI in the diagnosis of nutritional risk but had a good consistency with the combined assessment diagnosis of nutritional risk. See Tables 5,6.

\section{Discussion}

Chemotherapy is currently the primary treatment for preventing cancer metastasis and for metastatic middleto-advanced stage cancers. While killing cancer cells, chemotherapy drugs also damage rapidly proliferating normal cells, often leading to a series of adverse reactions, such as digestive tract damage, myelosuppression, and hair loss, etc. $(4,5)$. Therefore, it is critically important to evaluate the types of ADRs caused by chemotherapy and their related risk factors by screening the nutritional status of patients to maximize patient management before and after chemotherapy. The OPNI index is a simple and easy calculation using routine clinical measures and has good diagnostic efficiency in assessing nutritional risk. The NRS 2002 is a widely used current nutritional risk screening tool recommended by the Parenteral and enteral nutrition clinical diagnosis and treatment guidelines nutrition (2008). By combining both assessments, is an innovative attempt to guide the clinical.

This study retrospectively analyzed 199 patients with ADRs after cancer diagnosis and chemotherapy 
implementation. The difference in BMI between the OPNI and NRS 2002 subgroups was statistically significant, and the difference in ADR types in the OPNI group was statistically significant. The proportion of new and severe ADR types in the low OPNI group (47.14\%) was significantly higher than that in the high OPNI group $(27.13 \%)$. The differences in the OPNI, NRS 2002, and combined assessment groups in the two ADR categories of digestive tract injury and myelosuppression were statistically significant. Of the total ADR types, the incidence of digestive tract damage and myelosuppression was relatively high, with 27 cases and 130 cases, respectively. A logistic regression analysis was conducted for these two ADRs to evaluate the correlation between clinical variables and digestive tract damage or myelosuppression. We found that low BMI was a high-risk factor for both digestive tract damage and myelosuppressive ADRs after chemotherapy. We also analyzed the consistencies between NRS 2002 and OPNI, and NRS 2002 with the combined assessment. The analysis showed that the consistency between NRS 2002 and OPNI was not high, but the consistency between NRS 2002 and the combined assessment was relatively high. Cai et al. (2) believe that when the OPNI value is 45.8. Receiver operating characteristics of PNI and patient survival were plotted according to follow-up survival (ROC) curve to determine the optimal cutoff of PNI. it has a good consistency with NRS 2002, which is inconsistent with the conclusion of this study. We believe this may be related to the characteristics of the samples in this study. This study retrospectively analyzed a group of patients with ADRs after chemotherapy. OPNI, NRS 2002, and the combined use of OPNI and NRS 2002 scores were selected as the study samples for the screening of nutritional risk. Therefore, this study did not take nutritional risk as a functional category to predict the occurrence of ADRs. Instead, we studied the role of nutritional risk in assessing the type, classification, and factors related to ADRs.

Studies have shown that in patients receiving chemotherapy, the incidence of digestive tract damage is as high as $40-100 \%$, mainly presenting as oral ulcers, nausea, vomiting, abdominal pain, diarrhea, malnutrition, etc. Digestive tract damage not only seriously affects patients' quality of life, prolongs the length of hospital stay, and increases the cost of treatment but may also lead to treatment failure (6-8). The occurrence of this kind of adverse drug reaction may be related to the imbalance of intestinal flora caused by chemotherapy. Myelosuppression is one of the common chemotherapy-related dose-limiting side effects (9), including leukopenia, thrombocytopenia, neutropenia, etc. When considering chemotherapy drugs regimen involving chemotherapeutic agents suspected of causing digestive tract damage, such as those associated with a high risk of therapy-induced nausea and vomiting $(10,11)$ (cisplatin, carboplatin, cyclophosphamide, doxorubicin, etc.), and chemotherapeutic agents with an increased risk of myelosuppression (paclitaxel, fluorouracil, gemcitabine, doxorubicin, cyclophosphamide, etc.), treating physicians should consider the use of a combined nutritional risk screen to assess the nutritional status of patients prior to commencing chemotherapy. For patients identified as nutritionally at-risk after screening, their treating doctor would be able to foresee potentially serious ADRs and the increased risk of myelosuppression and digestive tract damage. According to the study, doctors can preventive intervention, reduce the occurrence of serious ADRs. Such as to improve the nutritional status of patients, especially in patients with chemotherapy regimens with high myelosuppression and high emetic risk. It is particularly important to improve the nutritional status of patients. In addition, the preventive use of some drugs, such as prevent use of anti-nausea drugs, prevent the use of G-CSF, etc.

Serious ADRs can lead to termination of chemotherapy, prolonged hospitalization time, increased hospitalization costs, and even life-threatening conditions. Therefore, preventive measures can be taken in advance to improve the nutritional status of patients and initiate the use of antiemetic drugs or G-CSF to ensure the success of the chemotherapy program (12-14).

In conclusion, NRS 2002 combined with OPNI can be used as a valuable method to evaluate the type and classification of ADRs in patients undergoing chemotherapy. Malnourished patients are more likely to develop serious ADRs during chemotherapy and have a higher incidence of digestive tract damage and myelosuppression. The use of NRS 2002 and NRS 2002 combined with OPNI showed good consistency. We believe the combined assessment is a better evaluation method to alert doctors to the need for early intervention measures in their patients and for pharmacists to carry out more rigorous pharmaceutical monitoring to improve patients' ability to tolerate chemotherapy and ensure the successful implementation of the treatment.

\section{Acknowledgments}

Funding: None. 


\section{Footnote}

Reporting Checklist: The authors have completed the STROBE reporting checklist. Available at https://atm. amegroups.com/article/view/10.21037/atm-22-256/rc

Data Sharing Statement: Available at https://atm.amegroups. com/article/view/10.21037/atm-22-256/dss

Conflicts of Interest: All authors have completed the ICMJE uniform disclosure form (available at https://atm. amegroups.com/article/view/10.21037/atm-22-256/coif). The authors have no conflicts of interest to declare.

Ethical Statement: The authors are accountable for all aspects of the work in ensuring that questions related to the accuracy or integrity of any part of the work are appropriately investigated and resolved. All procedures performed in this study involving human participants were in accordance with the Declaration of Helsinki (as revised in 2013). The study was approved by ethics board of Affiliated Hospital of Jiangnan University (No. LS2020034). Individual consent for this retrospective analysis was waived.

Open Access Statement: This is an Open Access article distributed in accordance with the Creative Commons Attribution-NonCommercial-NoDerivs 4.0 International License (CC BY-NC-ND 4.0), which permits the noncommercial replication and distribution of the article with the strict proviso that no changes or edits are made and the original work is properly cited (including links to both the formal publication through the relevant DOI and the license). See: https://creativecommons.org/licenses/by-nc-nd/4.0/.

\section{References}

1. Bray F, Ferlay J, Soerjomataram I, et al. Global cancer statistics 2018: GLOBOCAN estimates of incidence and mortality worldwide for 36 cancers in 185 countries. CA Cancer J Clin 2018;68:394-424.

2. Cai J, Weiqi H, et al. Prediction value of prognostic nutritional index for prognosis of patients with pancreatic head and periampullary carcinoma after laparoscopic pancreaticoduodenectomy. Chin J Hepat Surg(Electronic Edition)2020;9:441-2.

3. Adverse Drug Reaction Report and Monitoring Measures for The Administration. Available online: http://www.gov. cn/flfg/2011-05/24/content_1870110.htm
4. Hsu HC, Tsai SY, Wu SL, et al. Longitudinal perceptions of the side effects of chemotherapy in patients with gynecological cancer. Support Care Cancer 2017;25:3457-64.

5. García-Chías B, Figuero E, Castelo-Fernández B, et al. Prevalence of oral side effects of chemotherapy and its relationship with periodontal risk: a cross sectional study. Support Care Cancer 2019;27:3479-90.

6. Stringer AM. Interaction between host cells and microbes in chemotherapy-induced mucositis. Nutrients 2013;5:1488-99.

7. Thomsen M, Vitetta L. Adjunctive Treatments for the Prevention of Chemotherapy- and Radiotherapy-Induced Mucositis. Integr Cancer Ther 2018;17:1027-47.

8. Ali J, Khan AU, Shah FA, et al. Mucoprotective effects of Saikosaponin-A in 5-fluorouracil-induced intestinal mucositis in mice model. Life Sci 2019;239:116888.

9. Dale DC, McCarter GC, Crawford J, et al. Myelotoxicity and dose intensity of chemotherapy: reporting practices from randomized clinical trials. J Natl Compr Canc Netw 2003;1:440-54.

10. Jordan K, Jahn F, Aapro M. Recent developments in the prevention of chemotherapy-induced nausea and vomiting (CINV): a comprehensive review. Ann Oncol 2015;26:1081-90.

11. Cao J, Wang B, Wang Z, et al. Efficacy of mirtazapine in preventing delayed nausea and vomiting induced by highly emetogenic chemotherapy: an open label, randomized, multicenter phase III trial. J Clin Oncol 2018;36:1078.

12. Bohlius J, Herbst C, Reiser M, et al. Granulopoiesisstimulating factors to prevent adverse effects in the treatment of malignant lymphoma. Cochrane Database Syst Rev 2008;(4):CD003189.

13. Sung L, Nathan PC, Alibhai SM, et al. Meta-analysis: effect of prophylactic hematopoietic colony-stimulating factors on mortality and outcomes of infection. Ann Intern Med 2007;147:400-11.

14. Clark OA, Lyman GH, Castro AA, et al. Colonystimulating factors for chemotherapy induced febrile neutropenia: a meta analysis of randomized controlled trials. J Clin Oncol 2005;23:4198-214.

Cite this article as: Bian Y, Xie F, Han J, Ding Y. Nutritional evaluation study based on NRS 2002, OPNI, and their combined use in patients with adverse drug reactions after chemotherapy: a cross-sectional study. Ann Transl Med 2022;10(4):180. doi: 10.21037/atm-22-256 